CYBERNETICS AND INFORMATION TECHNOLOGIES • Volume 17, No 5

Special issue with selected papers from the workshop

"Two Years Avitohol: Advanced High Performance Computing Applications 2017"

Sofia $\bullet 2017$

Print ISSN: 1311-9702; Online ISSN: 1314-4081

DOI: $10.1515 /$ cait-2017-0058

\title{
Cloud Service for Numerical Calculations and Visualizations of Photonic Dissipative Systems
}

\author{
Hayk Grigoryan ${ }^{1}$, Hrachya Astsatryan ${ }^{1}$, Tigran Gevorgyan ${ }^{2,3}$, \\ Vahe Manukyan ${ }^{3}$ \\ ${ }^{1}$ Institute for Informatics and Automation Problems of the National Academy of Sciences of the Republic \\ of Armenia, 1, P. Sevak str., 0014 Yerevan, Armenia \\ ${ }^{2}$ Yerevan State University, 1 A. Manoogian st., 0015 Yerevan, Armenia \\ ${ }^{3}$ Institute for Physical Research of the National Academy of Sciences of the Republic of Armenia, \\ Ashtarak-2, 0203 Ashtarak Yerevan, Armenia \\ E-mails: haykgrigoryan@iiap.sci.am hrach@sci.am t_gevorgyan@ysu.am \\ manukyanvahe@gmail.com
}

\begin{abstract}
Nowadays quantum physics is crucial for several scientific applications, where it is no longer possible to neglect the environmental interaction, like dissipation and decoherence. In these cases, the quantum systems are usually treated as open systems and their time-evolution is described by a density matrix in frames of the master equation, instead of the Hilbert-space vector and the Schrodinger equation. The visualization of such quantum systems allows users to calculate and study the sensitivity of the parameters, like excitation photon numbers or photonnumber distribution functions or Wigner functions. In this paper, a cloud service for numerical calculations and visualization of photonic dissipative systems is presented, which enables numerical simulations and visualizations of a wide variety of Hamiltonians, including those with arbitrary time-dependences widely used in many physics applications. The service allows creating graphics and charts for interacting complex systems and simulating their time evolution with many available timeevolution drivers.
\end{abstract}

Keywords: Quantum optics, quantum simulations, visualization, Big Data, cloud service. 


\section{Introduction}

The quantum systems are usually treated as open systems and their time-evolution is described by a density matrix in the frame of the master equation, instead of the Hilbert-space vector and the Schrodinger equation. Therefore, in general, the master equation cannot be solved analytically for the most physical systems in arbitrary evaluation times and there is a need to use numerical methods. However, even a numerical solution of the master equation is a challenge. If a state vector $|\psi\rangle$ requires

$D$ basis vectors in Hilbert space to represent it, the corresponding density operator requires $D^{2}-1$ real numbers. This base requires large-scale simulations, especially when the system includes more than one degree of freedom. This problem can be solved by unraveling the density operator evolution into quantum trajectories and the Quantum State Diffusion (QSD) method [1]. Since quantum trajectories represent the system as a state vector rather than a density operator, they often have a numerical advantage over solving the master equation directly, even though one has to average over many quantum trajectories to recover the solution of the master equation. One of the widely used approximations for open quantum systems is the Markovian dynamics described in terms of the Lindblad master equation for the reduced density matrix $\rho$. In this case, the time-evolution is governed by the Hamiltonian operator $H$ and the Lindblad operators of the system. The QSD method has been implemented based on the stochastic equation that involves both Hamiltonian and the Lindblad operators for the state $|\psi(t)\rangle$. The density operator using an expansion of the state vector $|\psi\rangle$ in a truncated basis of Fock's number states of a harmonic oscillator (photonic states) is calculated.

The ECAPT (Extendible $\mathrm{C}^{++}$Application in Photonic Technologies) library and a web portal has been developed [2] providing numerical simulations and modeling of complex quantum systems in the presence of decoherence with a range of applications in photonics. Examples of those applications include investigation of quantum dissipative chaos, bistability and bifurcations [3, 4], quantum stochastic resonance [5], long-lived quantum interference in periodically modulated oscillatory systems [6], engineering of Fock states and qubits in nonlinear oscillators [7], elaboration of devices generating intensive entangled light beams for quantum communications [8], as well as production of three-photon entangled states in parametric devices [9], and numerical simulation of complex quantum optical systems, such as interaction of an atom with bichromatic laser field [10], cascaded processes [11] and photonics in ion-trap systems [12].

The aim of the paper is to extend the functionality of the portal by providing a cloud service for visualization and analytics to evaluate different physical dimensions of complex quantum systems. The service allows users to simulate and manage the results including the excitation photon numbers, probability, and Wigner functions, graphically for different parameters and time intervals. The rest of this paper is organized as follows: in Section 2 we introduce related work, Section 3 illustrates the scientific application based on Kerr nonlinear oscillator driven by chirped wave model, Section 4 represents the service description, and, finally, the use case is presented in Section 5 and conclusion in Section 6. 


\section{Related work}

Several projects that can be related to the data analyzes and visualization provide advanced functionalities but are typically restricted to a particular application area. They provide all the computational and visualization facilities needed to express and solve a target class of problems. Some examples of libraries and packages are of course Origin, Wolfram Mathematica or Gnuplot giving the abilities to perform builtin graph types and point-and-click customization of all elements.

There are a few projects based on the web providing advanced features for building data visualization environments to handle significant amounts of dynamic data, and to enable manipulation of and interaction with the data. They also supply facilities for deploying new services and provide APIs for generating web portals.

As there is no generic approach capable of handling complex quantum systems, a cloud service is suggested aiming to simplify the use of visualization libraries deployed as a set of services.

The globalization of resources (hardware and software) on a large scale distributed computing infrastructures requires advanced trading mechanisms to identify the service or the composition of services able to fulfill a user request. The service may use high-performance computational resources without being aware of the locations of input and output data, as well as computational resources. This is crucial, as the usage of distributed computing infrastructures are complex and make difficulties for scientists and researchers to use the infrastructure without advanced knowledge and experience. Therefore the suggested service provides a web-based visualization with dynamic, browser-based visualization libraries making easy for a scientist to use the service without installing any extra tools for data visualization.

\section{Kerr nonlinear oscillator driven by chirped wave model}

In this section, the chirped Kerr-nonlinear resonator in a regime of continuous-wave driving scientific application is presented on the base of the master equation in the complete quantum analysis of autoresonant transitions and phase locking phenomena in the presence of dissipation. This approach allows us to consider control of a production of photon-number states and enhancement of photon blockade by selfadjusting the drive frequency of resonator. In the Photon Blockade (PB) based on photon-photon interaction in a Kerr-nonlinear element, the optical response to a single photon is modulated by the presence or absence of the other photons. Mainly, the capture of a single photon into the system affects the probability that a second photon is admitted. A simple consequence of photon blockade is the antibunching of photons in emission in analogy to the photon antibunching of resonance fluorescence on a two-level atom $[13,14]$. Photon blockade was first observed in an optical cavity coupled to a single trapped atom [15]. The PB has been predicted in cavity Quantum ElectroDynamics (QED) [16], and recently in circuit QED with a single superconducting artificial atom coupled to a microwave transmission line resonator $[17,18]$. PB was also experimentally demonstrated with a photonic crystal cavity with a strongly coupled quantum dot [19], and was also predicted in quantum 
optomechanical systems [20, 21]. An analogous phenomenon of phonon blockade was predicted for an artificial superconducting atom coupled to a nanomechanical resonator [22], as well as the polariton blockade effect due to polariton-polariton interactions has been considered in [23]. Recently, PB was considered in dispersive qubit-field interactions in a superconductive coplanar waveguide cavity [17] and with time-modulated input [24]. We clarify the chirp effects in Kerr nonlinear resonator by considering photon-number effects and by analysing phase-space properties of resonator mode. Thus, we focus on the analysis of the mean photon number (excitation number), the probability distributions of photons and the Wigner functions in phase space.

We treat the chirped Kerr-nonlinear resonator as an open quantum system and assume that it's time evolution is described by Markovian dynamics in terms of the Lindblad master equation for the reduced density matrix. In the interaction picture that corresponds to the transformation $\rho \rightarrow e^{-i \omega a^{+} a t} \rho e^{i \omega a^{+} a t}$, where $a^{+}$and $a$ are the Bose creation and annihilation operators of the oscillatory mode and is the driving frequency, this equation reads in the Markovian form as

$$
\frac{d \rho}{d t}=\frac{-i}{\hbar}[H, \rho]+\sum_{i=1,2}\left(L_{i} \rho L_{i}^{+}-\frac{1}{2} L_{i}^{+} L_{i} \rho-\frac{1}{2} \rho L_{i}^{+} L_{i}\right) .
$$

The Hamiltonian of Kerr-nonlinear resonator under pulsed excitation in the rotating wave approximation reads as:

$$
H=(\Delta-\delta t) a^{+} a+\chi\left(a^{+}\right)^{2} a^{2}+\left(\Omega a^{+}+\Omega^{*} a\right) .
$$

Here, time-dependent coupling constant $\Omega$ that is proportional to the amplitude of the driving field while $a+, a$ are the Bose creation and annihilation operators, $\chi$ is the nonlinearity strength, and $\Delta=\omega_{0}-\omega$ is the detuning between the mean frequency of the driving field and the frequency of the oscillator, $\delta$ is the chirp parameter. The dissipative and decoherence effects, losses, and thermal noise are included in the last part of this equation, where $L_{i}$ are the Lindblad operators:

$$
L_{1}=\sqrt{(N+1) \gamma} a \quad L_{2}=\sqrt{N \gamma} a^{+},
$$

$\gamma$ is the spontaneous decay rate of the dissipation process and $N$ denotes the mean number of quanta of a heat bath.

This model seems experimentally feasible and can be realized in several physical systems. Particularly, the effective Hamiltonian (Equation (2)) describes a qubit off-resonantly coupled to a driven cavity. In fact, it is well known that the Hamiltonian of a two-level atom interacting with cavity mode in the dispersive approximation, if the two-level system remains in its ground state, can be reduced to the effective Hamiltonian (Equation (2)). This model also describes a nanomechanical oscillator with $a^{+}$and $a$ raising and lowering operators related to the position and momentum operators of a mode quantum motion. An important implementation of the Kerr-type resonator has been recently achieved in the context of superconducting devices based on the nonlinearity of the Josephson junction.

We analyze the master equation numerically using quantum state diffusion method QSD [1]. According to this approach, the reduced density operator is 
calculated as the ensemble mean

$$
\rho(t)=M\left(\left|\psi_{\xi}(t)\right\rangle\left\langle\psi_{\xi}(t)\right|\right)=\lim _{N \rightarrow \infty} \frac{1}{N} \sum_{\xi}^{N}\left|\psi_{\xi}(t)\right\rangle\left\langle\psi_{\xi}(t)\right|,
$$

over the stochastic pure states $\left|\psi_{\xi}(t)\right\rangle$ describing evolution along a quantum trajectory. The stochastic equation for the state $\left|\psi_{\xi}(t)\right\rangle$ involves both Hamiltonian described by Equation (2) and the Linblad operators described by Equation (3). We calculate the density operator using an expansion of the state vector $\left|\psi_{\xi}\right\rangle$ in a truncated basis of Fock's number states of a harmonic oscillator,

$$
\left|\psi_{\xi}(t)\right\rangle=\sum_{n} a_{n}^{\xi}(t)|n\rangle .
$$

The photon-number distribution is calculated as the diagonal elements of the photon-number states $|n\rangle$

$$
P(n)=\langle n|\rho| n\rangle,
$$

while calculations of the Wigner function are performed by using its standard form in a Fock space:

$$
W(\rho, \theta)=\sum_{m, n} \rho_{m n} W_{m n}(\rho, \theta) .
$$

Here, $\rho, \theta$ are the polar coordinates in the complex phase space, which is determined by position and momentum of the quadratures $x=\left(a+a^{+}\right) / \sqrt{2}, \quad y=\left(a-a^{+}\right) / \sqrt{2} i$, respectively, while the coefficients $W_{m n}(\rho, \theta)$ are the Fourier transforms of the matrix elements of the Wigner characteristic function.

\section{Service description}

The architecture of the service consists of a front-end, server and the REST (REpresentational State Transfer) API components (Fig. 1). All the difficulties inherent in the use of cloud APIs, visualization libraries and MPI programming are hidden to the user which is the main goal of our generic approach.

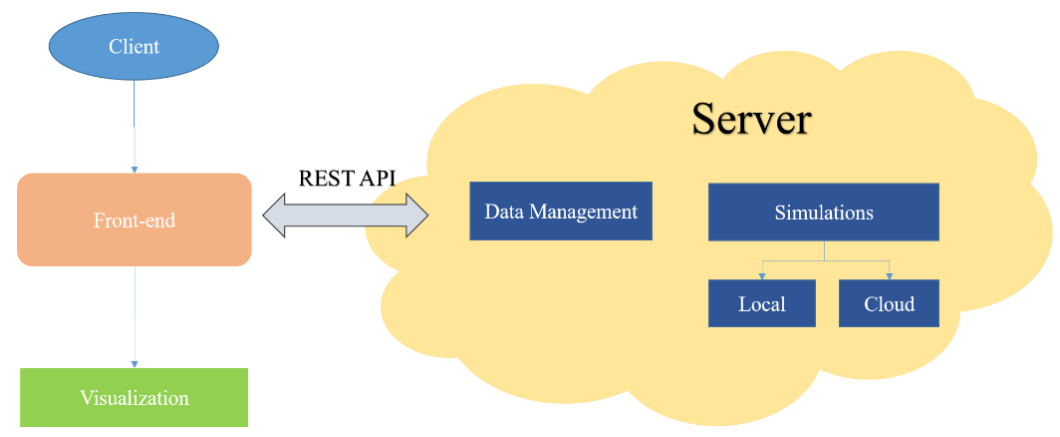

Fig. 1. Cloud service architecture for numerical calculations and visualizations of photonic dissipative systems 
Front-end is a web-based, service-rich environment for the development, execution and monitoring workflow based parameter studies on various grid platforms photonic dissipative systems. Front-end hides low-level cloud access mechanisms by high-level graphical interfaces; making even non-cloud expert users capable of defining and executing photonic dissipative systems on distributed computing infrastructures. It allows users both to manipulate (initialize, upload, suppress) the data and to submit the computational and visualization requests using the input parameters for simulations. The visualization layer is responsible for interactive visualization of the data of the user submitted simulations results.

Server component is based on a module for the simulation and data management. Data Management consists of the file system and the MySQL database. The simulation layer executes the simulation on a local resource or HPC resources taking into account the complexity of the application service.

The REST API is a middleware for handling the requests and organizing the communication between front-end and the server. It stands for is an architectural style providing interoperability between computer systems on the Internet.

\subsection{Front-end}

The cloud service is based on user authentication capabilities, providing a userfriendly environment for constructing and executing the simulations. The platform provides an ability to calculate excitation photon numbers, probability function and Wigner functions with different input parameters. For Hamiltonian of Kerr nonlinear resonator under pulsed excitation in the rotating wave approximation (Equation (2)):

- The time dependent coupling constant $\Omega$.

- The nonlinearity strength $\chi$.

- The detuning $\Delta=\omega_{0}-\omega$ between the mean frequency of the driving field and the frequency of the oscillator.

- The chirp parameter $\delta$ and the numerical calculation parameters.

- The time interval from 0 to $t$.

- The calculation time step $d t$.

- The state vector size in a truncated basis of Fock's states bases.

After submitting new simulations, the front-end sends commands to the REST API. As soon as the calculation completes, the results, including the needed parameters are available in the front-end part, after which the front-end sent the request by asking for similar results and after getting already constructed response required for visualization module, it displays the visualization. Based on the simulation results the D3.js [25] and Vis.js [26] are used for visualization correspondingly for line charts and 3D function visualization. The Front-end platform has been implemented with Angular JS framework.

\subsection{Data management}

Two types of datasets are used for visualization. One is two-dimensional excitation photon numbers, probability functions stored in an ASCII file, which introduces the evaluation in a time interval. There are two double numbers separated by a blank space per each line. First one is the time and the second one is the corresponding 
value. The second type is the three-dimensional Wigner function result also stored in an ASCII file. Each line contains three doubles $(x, y, z)$ separated with spaces.

For storing users and the simulations history, the MySQL database is used to synchronize with the file system. The table called "results" contains information about each simulation. For each one, it stores the initial input parameters for ECAQT program and the status of that simulation. For each simulation in the file system platform creates a working directory.

\subsection{Simulations}

During the service flow, the cron job reads a new submitted simulation from the database and for each one, depending on the complexity of the simulation, either runs the ECAQT program in the local environment or sends the task to the cloud service for execution. During the simulations, it creates an excitation photon number, probability function and the Wigner functions which are stored in the text files. For visualization, the program reads text files containing functions and parses it for visualization.

\subsection{User interface}

The Web portal provides a flexible and easy to use interface for a scientist to make simulations and do their analysis. Users can SingIn/SignUp to the web portal by clicking buttons on the top right corner.

After entering the system, the user will navigate the main dashboard screen. Here the user can submit the new simulations by choosing the different input parameters by filling the necessary input parameters in the top form and clicking to the "Submit" button (Fig. 2).

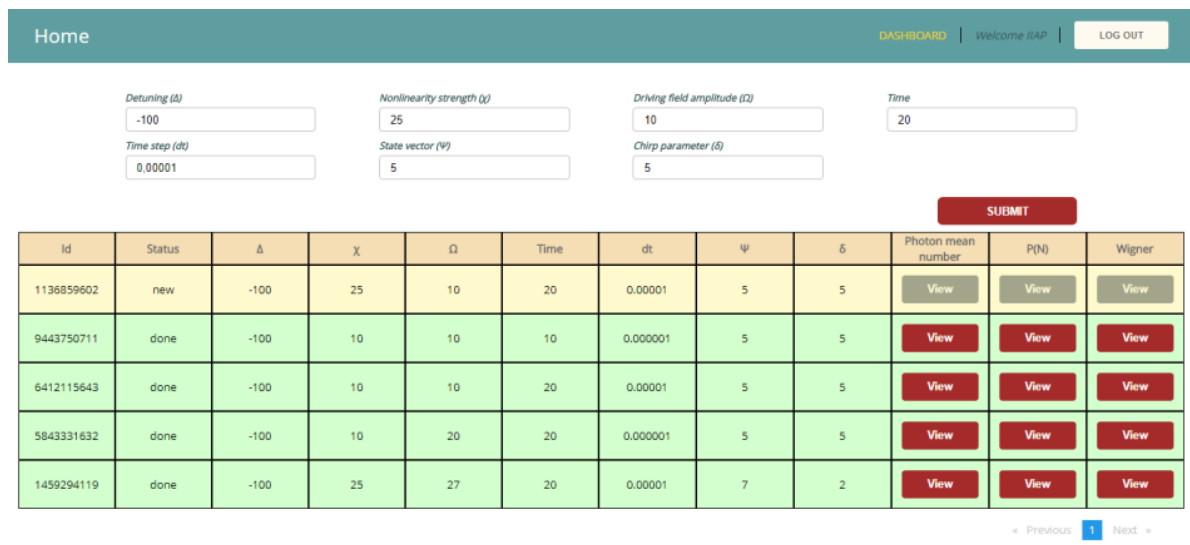

Fig. 2. Service dashboard page

The table illustrates the history of simulations. When the simulation is finished successfully, the user can see visualizations by clicking on the "View" buttons on that row.

- Photon mean number $\langle n\rangle$ (see Fig. 3).

- Photon-number distribution functions $P(n), n=0, \ldots, 4$ (see Fig. 5). 
- Wigner function for given $t$ and Wigner function evolution (see Fig. 6).

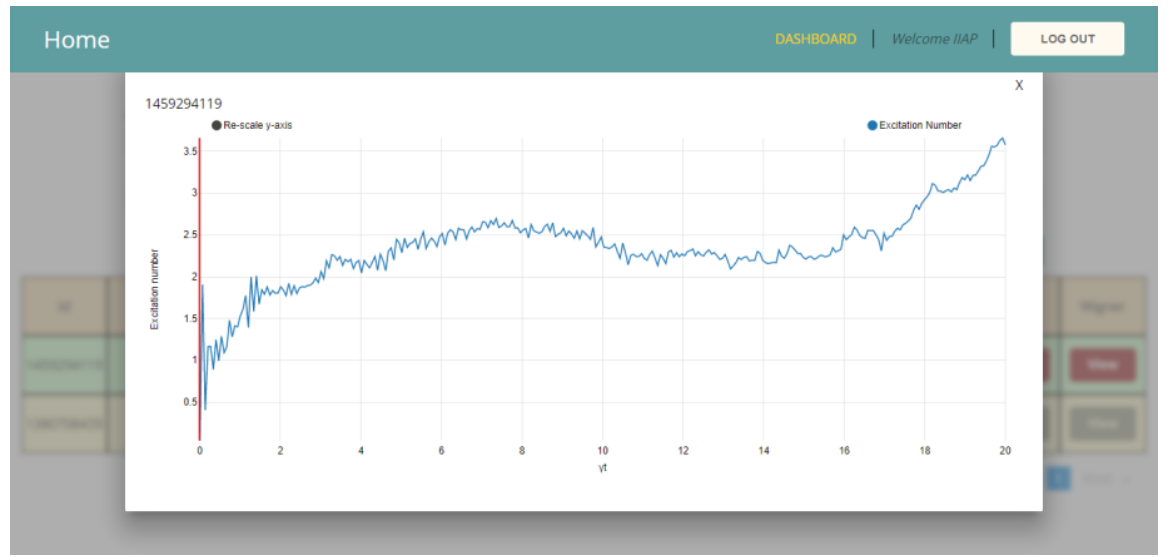

Fig. 3. Excitation number visualization chart

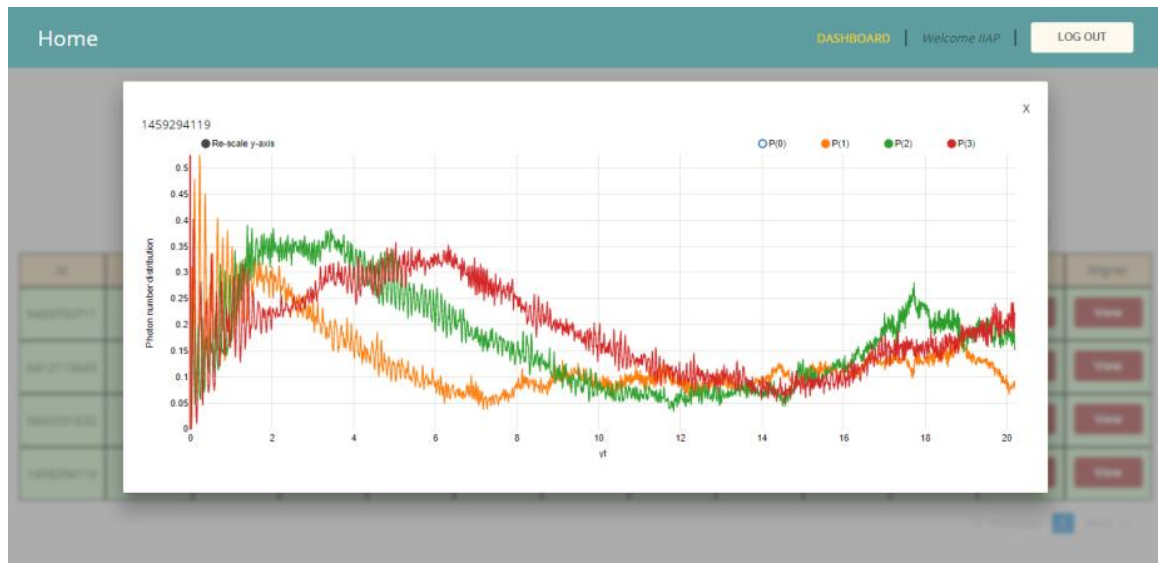

Fig. 4. Photon-number distribution function visualization chart

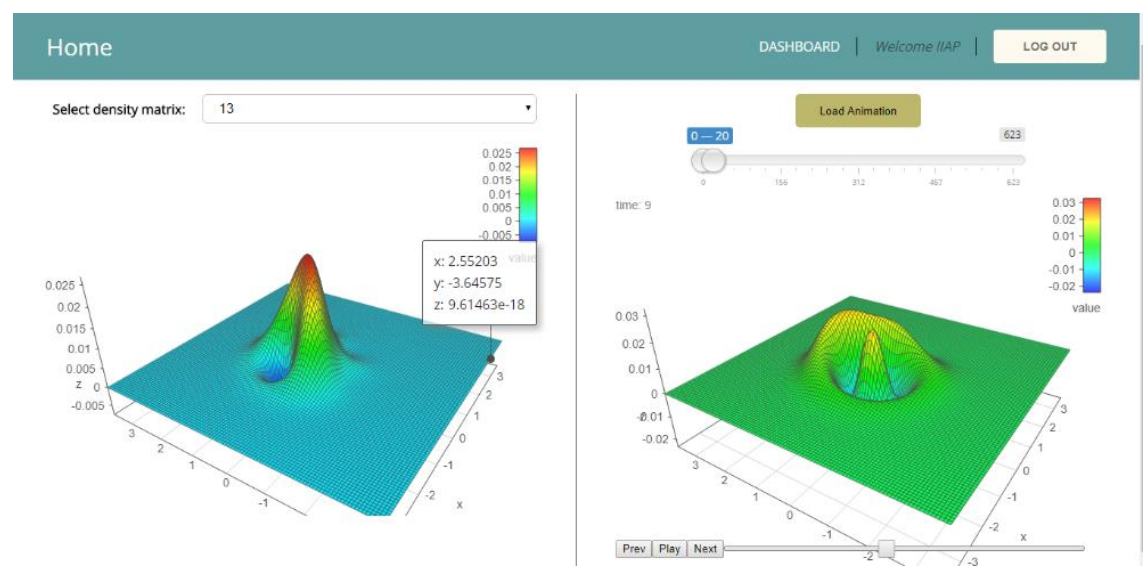

Fig. 5. Screenshot of the Wigner function visualization 
The Wigner function visualization screen is separated into two sections: (a) and (b). Section (a) is the single 3D function, where a user can select the time for what the visualization is needed. In Section (b), user can choose the time range and see the evolution of the changes of the Wigner functions with excellent animation. Visualizations are interactive so the user can rotate, zoom in/out and by selecting the particular point in the graph can see the $(x, y, z)$ values.

\section{Use case}

The possibility of photon blockade realization with the use of a chirped dissipative field represents a significant interest nowadays.

We have performed various calculations with the utilisation of this tool to study the described system. Fig. 6 shows time dynamics of the photon state probabilities. The dynamics of the external field with no chirp is illustrated in Fig. 6a, while Fig. $6 \mathrm{~b}$ demonstrates the dynamics when chirp is applied. It can be seen from Fig. 6b that a state with significant probability is generated for small nonlinearities $(\chi / \gamma=3)$ and for the field amplitude $\Omega / \gamma=2.5$.

Let us now discuss the state illustrated in Fig. $6 \mathrm{~b}$ when the $P_{1}$ and the $P_{0}$ states of the two different time moments have equal probabilities of 0.5 value, and therefore, it is possible to produce superposition states for these time moments. We check the existence of superposition states with the use fidelity that shows the closeness of two quantum states. Thus we calculate it for the $|\psi\rangle=\frac{1}{\sqrt{2}}(|0\rangle-|1\rangle)$ superposition state and for the states corresponding to the crossing points. Our calculations have shown that this fidelity for those moments is 0.78 , which indicates that the condition obtained is very close to a pure superposition state. The similarity of these two states can also be demonstrated using the Wigner function. The Wigner functions for $\frac{1}{\sqrt{2}}(|0\rangle-|1\rangle)$ state and for the given time moment are represented in Fig. 7a and b, respectively. As we can see from the picture, the Wigner functions are very close, however, an obvious difference can be observed for the negative parts of the Wigner functions. In contrast to the pure state, the negative part of the generated state is smaller. It is known that the negative part of the Wigner function indicates whether the given state is a pure quantum state. The small absolute value of the function's negative part can be explained by the presence of decoherence and dissipation as these effects eliminate the quantum state.

The case demonstrated in Fig. 6a is a well-studied case when the external field that affects on the quantum nonlinear oscillator is monochromatic, and the presence of the dissipation is taken into account. As we can see, there are several excitations at the start point that shrink with time and eventually, the system comes to a steady state. As we can see from Fig. $6 \mathrm{~b}$ the situation is entirely different when the chirp is applied. There are only small oscillations at the start point, and the energy is localized at a specific moment of time. At this time the single-photon state is obtained. 

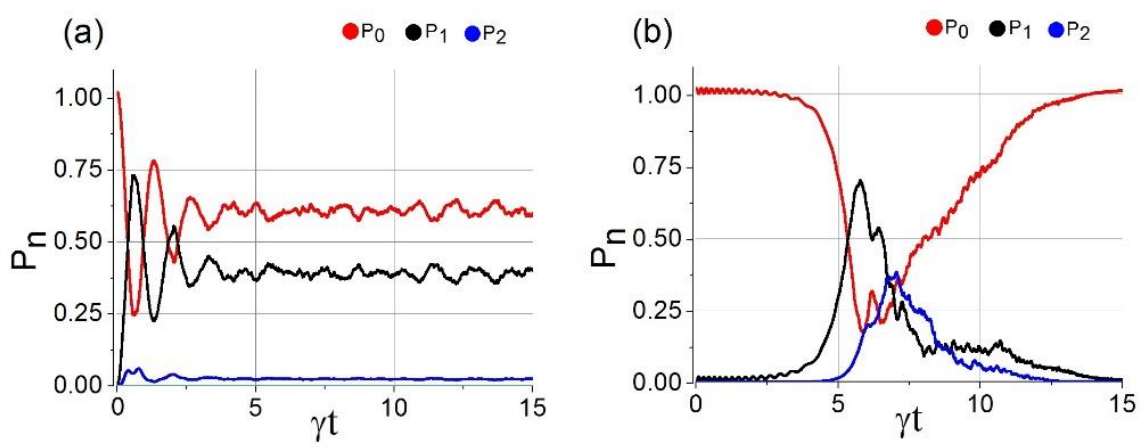

Fig. 6. Wigner functions for the case of external chirped field: (a) for the state $\frac{1}{\sqrt{2}}(|0\rangle-|1\rangle)$; (b) for the chirped field. The parameters are as follows: (a) $\chi / \gamma=5, \Omega / \gamma=2.5, \alpha / \gamma^{2}=0$; (b) $\chi / \gamma=5$, $\Omega / \gamma=2.5, \alpha / \gamma^{2}=7$
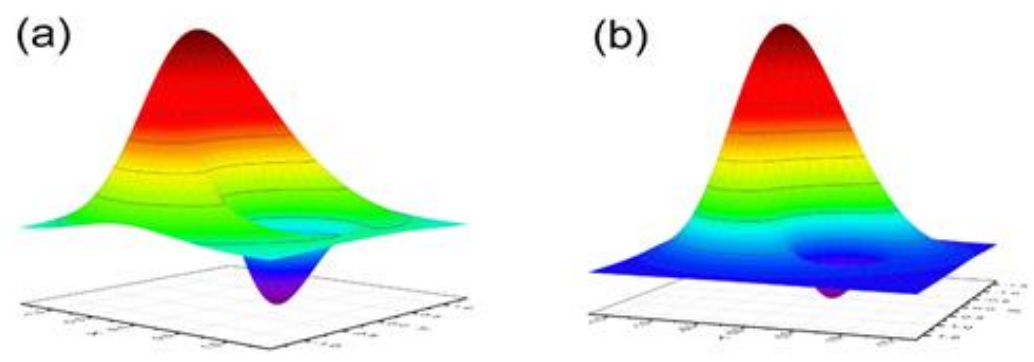

Fig. 7. Wigner functions for the case of external chirped field: (a) for the state $\frac{1}{\sqrt{2}}(|0\rangle-|1\rangle)$; (b) for the chirped field. The parameters are as follows: (a) $\chi / \gamma=5, \Omega / \gamma=2.5, \alpha / \gamma^{2}=0$, (b) $\chi / \gamma=5$, $\Omega / \gamma=2.5, \alpha / \gamma^{2}=7$

\section{Conclusion}

The visualization of the results (Figs 3-5) help researchers to analyze and study the systems more practically and more completely for different time intervals and time points, for instance to analyze the output results for different input parameters for system Hamiltonian (Equation (2)) and for different values of same Hamiltonian for different times. Besides providing storage for different simulation results, we make theoretical research more experimental and visual saving research time and resources. The ultimate goal is to develop a single sign-on integrated multi-service environment providing easy access to different kind of quantum calculations and algorithms to be performed on hybrid distributed computing infrastructures combining the benefits of large clusters, grid or cloud, when needed.

We have demonstrated the production of single photon blockade for frequencychirped fields. 
Acknowledgments: The research leading to these results has been co-funded by the European Commission under the H2020 Research Infrastructures contract No 675121 (Project VI-SEEM) and the Armenian State Committee of Science under the Projects 15T-1B292, No 15T-1C052.

\section{References}

1. G i s i n, N., I. C. P e r c i v a 1. The - Quantum-State Diffusion Model Applied to Open Systems. J. Phys., Vol. A25, 1992, 5677; I. C. Percival, Quantum State Diffusion. Campridge, Cambridge University Press, 2000. Doi:10.1088/0305-4470/25/21/023.

2. A st satry a n, H., T. Gevorgy a n, A. Sh ah in y a n. Web Portal for Photonic Technologies Using Grid Infrastructures. - Journal of Software Engineering and Applications, Vol. 5, 2012, No 11, pp. 864-869, Doi: 10.4236/jsea.2012.511100.

3. Kryuchkyan, G. Yu, S. B. Manvely a n. Quantum Dissipative Chaos in the Statistics of Excitation Numbers. - Phys. Rev. Lett., Vol. 88, 2002, 094101. Doi:10.1103/PhysRevLett.88.094101.

4. Kryuchkyan, G. Yu., S. B. Manvelyan. Sub-Poissonian Statistics in Order-to-Chaos Transition. - Phys. Rev., Vol. A68, 2003, 013823. Doi:10.1103/PhysRevA.68.013823.

5. A d a my a n, H. H., S. B. Manvely a n, G. Y u. Kry u ch ky a n. Stochastic Resonance in Quantum Trajectories for an Anharmonic Oscillator. - Phys. Rev., Vol. A63, 2001, 022102. Doi:10.1103/PhysRevA.63.022102.

6. G e vorg y a n, V., A. R. S h a h i n y a n, G. Y u. K r y u c h k y a n. Quantum Interference and SubPoissonian Statistics for Time-Modulated Driven Dissipative Nonlinear Oscillators. - Phys. Rev., Vol. A79, 2009, 053828. Doi:10.1103/PhysRevA.79.053828.

7. Gevorgy a n, T. V., A. R. Shah in y a n, G. Yu. Kry u ch ky a n. Generation of Fock States and Qubits in Periodically Pulsed Nonlinear Oscillators. - Phys. Rev., Vol. A85, 2012, 053802. Doi:10.1103/PhysRevA.85.053802.

8. A d a my a n, H. H., N. H. Ad a my a n, S. B. Manvely an, G. Yu. Kryuchkyan. Quadrature Entanglement and Photon-Number Correlations Accompanied by Phase-Locking. - Phys. Rev., Vol. A73, 2006, 033810.

9. A n to nos y a n, D. A., T. V. Gevorgy a n, G. Yu. Kry u ch k y a n. Three-Photon States in Nonlinear Crystal Superlattices. - Phys. Rev., Vol. A83, 2011, 043807. Doi:10.1103/PhysRevA.83.043807.

10. J a k o b, M., G. Y u. K r y u c h k y a n. Autler-Townes Effect with Mono- and Bichromatic-Pump Fields: Reservoir Effects and Floquet-State Treatment. - Phys. Rev., Vol. A57, 1998, 1355. Doi:10.1103/PhysRevA.57.1355.

11. Kheruntsyan, V., G. Yu. Kryuchky an, N. T. Mouradyan, K. G. Petrossian. Controlling Instability and Squeezing from a Cascaded Frequency Doubler. - Phys. Rev., Vol. A57, 1998, 535. Doi:10.1103/PhysRevA.57.535.

12. J a k o b, M., G. Y u. K r y u c h k y a n. Photon Correlation in an Ion-Trap System. - Phys. Rev., Vol. A59, 1999, 2111. Doi:10.1103/PhysRevA.59.2111.

13. Carmich a e 1, H. J., D. F. W a $11 \mathrm{~s}$. Intensity Correlations in Resonance Fluorescence with Atomic Number Fluctuations. - J. Phys. B: Atom. Molec. Phys., Vol. L43, 1976, No 9.

14. K i m b l e, H. J., M. D a g e $\mathrm{n}$ a i s, L. M a n d e l. Photon Antibunching in Resonance Fluorescence. - Phys. Rev. Lett., Vol. 39, 1977, 691.

15. B i r n b a u m, K. M., et al. Photon Blockade in an Optical Cavity with One Trapped Atom. - Nature, Vol. 436, 2005, 87.

16. Ti a n, L., H. J. Carmi c h a e 1. Quantum Trajectory Simulations of Two-State Behavior in an Optical Cavity Containing One Atom. - Phys. Rev., Vol. A46, 1992, R6801.

17. H o f f m a n, A. J., et al. Search for Dilepton Resonances in pp Collisions at $\mathrm{s}=7 \mathrm{TeV}$ with the ATLAS Detector. - Phys. Rev. Lett., Vol. 107, 2011, 053602.

18. Lang, C., et al. Observation of Resonant Photon Blockade at Microwave Frequencies Using Correlation Function Measurements. - Phys. Rev. Lett., Vol. 106, 2011, 243601. 
19. F a r a o n, A., I. F u s h m a n, D. En g l u n d, N. S t o l t z, P. P e t r of f, J. V u c k o vi c. Coherent Generation of Non-Classical Light on a Chip via Photon-Induced Tunnelling and Blockade. Nat. Phys., Vol. 4, 2008, pp. 859-863.

20. R a b 1, P. Photon Blockade Effect in Optomechanical Systems. - Phys. Rev. Lett., Vol. 107, 2011, 063601.

21. N u n n e n k a m p, A., K. Bør k j e, S. M. G i r vi n. Single-Photon Optomechanics. - Phys. Rev. Lett., Vol. 107, 2011, 063602.

22. Liu, Y. X., A. Miranowicz, Y. B. Gao, J. B ajer, C. P. Sun, F. Nori. Testing Nonclassicality in Multimode Fields: A Unified Derivation of Classical Inequalities. - Phys. Rev., Vol. A82, 2010, 032101.

23. V e r g e r, A., C. C i u t i, I. C a r u s o t t o. Polariton Quantum Blockade in a Photonic Dot. - Phys. Rev., Vol. B73, 2006, 193306.

24. Far a o n, A., A. M a j u m d a r, J. V u c k o vi c. Generation of Nonclassical States of Light via Photon Blockade in Optical Nanocavities. - Phys. Rev., Vol. A81, 2010, 033838.

25. A b h i j i t, J. Data Visualization with the D3. JS JavaScript library. - Journal of Computing Sciences in Colleges, Vol. 30, 2014, No 2, pp. 139-141.

26. S c o t t, W. Adding Visualization with JSON. Pro j Query in Oracle Application Express. Apress, 2015, pp. 133-147. 